\title{
Classical Models of Confinement II
}

\author{
Harry Lehmann ${ }^{\star}$ and Tai Tsun $\mathrm{Wu}{ }^{\star \star}$ \\ 1 II. Institut für Theoretische Physik der Universität Hamburg, D-2000 Hamburg, \\ Federal Republic of Germany \\ 2 Deutsches Elektronen-Synchrotron DESY, D-2000 Hamburg, Federal Republic of Germany, \\ and Gordon McKay Laboratory, Harvard University, Cambridge, MA 02138, USA
}

\section{Dedicated to the memory of Kurt Symanzik}

\begin{abstract}
We generalize the class of Abelian models of paper I which lead to a linear potential between opposite charges. The electric field $E$ is here taken as a power series in the electric displacement $D$ raised to the $\sigma^{\text {th }}$ power. We solve to first order the case of two opposite static point charges asymptotically for large separations.
\end{abstract}

\section{Introduction}

In a recent paper [1] (hereafter referred to as I), we investigated classical models of confinement. These models are essentially those previously studied by Pagels and Tomboulis [2] and more extensively by Adler and Piran [3]. They can be viewed as ordinary electrostatics with a field-dependent dielectric constant.

In I we considered the case

$$
E=f(D)=E_{0}+b_{1} D+b_{2} D^{2}+\ldots,
$$

with $b_{1}>0$. Without loss of generality we can set $E_{0}=b_{1}=1$. For two unit static charges of opposite sign, separated by a large distance $2 R$, it was found that the flux is confined within an ellipsoid of revolution with semi-minor axis $(2 R / \sqrt{\pi})^{1 / 2}$, leading to a linear potential with a correction of order $\ln R$. We also studied briefly the more general case,

$$
E=f(D)=1+D^{\sigma}+\ldots,
$$

with $\sigma>0$ and we showed that the transverse dimension of the confinement domain increases as $R^{1 /(1+\sigma)}$ for large $R$.

It is the purpose of the present note to examine further the case (1.2). This more general case is of interest in view of the uncertainty in the shape of the confinement domain for a realistic theory such as QCD.

* Work supported in part by NATO Research Grant No. 432/84

$\star \star$ Work supported in part by the U.S. Department of Energy under Grant No. DE-FG02-84ER40158 with Harvard University 


\section{Zeroth-Order Solution}

With the charges located at $\pm R$ along the $z$-axis of a cylindrical coordinate system, we can express $D$ in terms of a flux function $\Psi(z, \varrho)$ as [3],

$$
\varrho D_{z}=\frac{1}{2 \pi} \partial_{\varrho} \Psi, \quad \varrho D_{\varrho}=-\frac{1}{2 \pi} \partial_{z} \Psi .
$$

This $\Psi$ satisfies the partial differential equation [Eq. (2.11) of I]

$$
\partial_{z}\left[\frac{\partial_{z} \Psi f(D)}{\left[\left(\partial_{z} \Psi\right)^{2}+\left(\partial_{\varrho} \Psi\right)^{2}\right]^{1 / 2}}\right]+\partial_{\varrho}\left[\frac{\partial_{\varrho} \Psi f(D)}{\left[\left(\partial_{z} \Psi\right)^{2}+\left(\partial_{\varrho} \Psi\right)^{2}\right]^{1 / 2}}\right]=0,
$$

with the boundary conditions given by (2.10) of I. If we express $\varrho$ as a function of $z$ and $\Psi$, viz.,

$$
\varrho=\varrho(z, \Psi),
$$

(2.2) becomes

$$
\frac{-g(D) \partial_{z z} \varrho \cdot \operatorname{sgn}\left(\partial_{\Psi} \varrho\right)}{\left[1+\left(\partial_{z} \varrho\right)^{2}\right]^{3 / 2}}+\frac{1}{2 \pi}\left[\frac{1}{\partial_{\Psi} \varrho} \partial_{\Psi} \frac{1}{\varrho \partial_{\Psi} \varrho}+\frac{\partial_{z} \varrho}{\partial_{\Psi} \varrho} \partial_{\Psi} \frac{\partial_{z} \varrho}{\varrho \partial_{\Psi} \varrho}-\partial_{z} \frac{\partial_{z} \varrho}{\varrho \partial_{\Psi} \varrho}\right]=0,
$$

where

$$
g(D)=\frac{f(D)}{f^{\prime}(D)}-D
$$

In the limit of large $R$, the weak field approximation $f(D)=1+D^{\sigma}$ and, hence,

$$
g(D)=\sigma^{-1} D^{1-\sigma}
$$

can be applied far away from the charges. In this region, $\partial_{\Psi} \varrho<0$ and (2.4) simplifies to zeroth-order in $R$ :

$$
\partial_{z z} \varrho-\frac{1}{(2 \pi)^{\sigma}} \cdot \frac{1}{\partial_{\Psi} \varrho} \partial_{\Psi}\left(\frac{-1}{\varrho \partial_{\Psi} \varrho}\right)^{\sigma}=0 .
$$

The separation of variables

$$
\varrho(z, \Psi)=Z(z) G(\Psi),
$$

leads to the results

$$
z=\frac{2 R}{\sqrt{\pi}} \frac{\Gamma\left(\frac{1}{2 \sigma}+1\right)}{\Gamma\left(\frac{1}{2 \sigma}+\frac{1}{2}\right)} \sqrt{1-Z^{2 \sigma}} F\left(-\frac{1}{2 \sigma}+\frac{1}{2}, \frac{1}{2} ; \frac{3}{2} ; 1-Z^{2 \sigma}\right),
$$

and

$$
G(\Psi)=(\pi \sigma)^{-1 / 2}\left(\sqrt{2} R(\sigma+1)^{\sigma / 2} \frac{\Gamma\left(\frac{1}{2 \sigma}\right)}{\Gamma\left(\frac{1}{2 \sigma}+\frac{1}{2}\right)}\right)^{\frac{1}{\sigma+1}}\left[1-\Psi^{\frac{\sigma}{\sigma+1}}\right]^{1 / 2}
$$


where $F$ is the hypergeometric function. For $\sigma=1 / n(n=1,2,3, \ldots)$ this hypergeometric function is elementary.

It follows from this explicit solution that

$$
\Psi(z, \varrho)=\left(1-\frac{\bar{\varrho}^{2}}{Z^{2}(z)}\right)^{\frac{\sigma+1}{\sigma}},
$$

where $\varrho$ is related to $\varrho$ by

$$
\varrho=(\pi \sigma)^{-1 / 2}\left(\sqrt{2} R(\sigma+1)^{\sigma / 2} \frac{\Gamma\left(\frac{1}{2 \sigma}\right)}{\Gamma\left(\frac{1}{2 \sigma}+\frac{1}{2}\right)}\right)^{\frac{1}{\sigma+1}} \bar{\varrho} .
$$

The boundary $B$ of the confinement domain is accordingly given by

$$
\bar{\varrho}_{b}=Z(z) \text {. }
$$

Note in particular that, when $z=0, Z=1$ and hence the maximal radius of the confinement domain is given by (2.12) with $\varrho=1$.

\section{Electrostatic Energy}

The total electrostatic energy $V(R)$ is given by

$$
V(R)=\int d^{3} x \int_{0}^{D} d D^{\prime} f\left(D^{\prime}\right) \sim \int d^{3} x\left(D+\frac{1}{\sigma+1} D^{\sigma+1}\right) .
$$

The substitution of the zeroth-order solution (2.11) into (3.1) leads to

$$
V(R)=2 R+\frac{2(1+\sigma)}{(1+2 \sigma)(1-\sigma)}\left(\frac{1+\sigma}{2}\right)^{\frac{\sigma}{\sigma+1}}\left(\frac{\Gamma\left(\frac{1}{2 \sigma}+\frac{1}{2}\right)}{\Gamma\left(\frac{1}{2 \sigma}\right)}\right)^{\frac{2 \sigma}{1+\sigma}} R^{\frac{1-\sigma}{1+\sigma}} .
$$

When $\sigma \rightarrow 1$, this agrees with (4.17) of I. When $\sigma>1$, a divergent integral is encountered which has been interpreted in the sense of distributions [4]. With a suitable choice of an additive constant in $V(R),(3.2)$ gives the leading correction to the linear potential.

\section{Reformulation of the Problem}

In (2.11) we have exhibited the zeroth-order of (2.2) for large $R$. To go beyond this zeroth-order in the present case of general $\sigma$, the choice of variables is important. The underlying reason is that the zeroth-order solution already involves a transcendental function, as seen from (2.9). In the remainder of this paper, we shall consider the case $f(D)=1+D^{\sigma}+b_{2} D^{2 \sigma}+\ldots$.

We generalize the procedure of Sect. 5.2 of I. First we transform from $z$ to a variable $\zeta$ by

$$
z=\frac{\zeta}{\beta} F\left(-\frac{1}{2 \sigma}+\frac{1}{2}, \frac{1}{2} ; \frac{3}{2} ; \zeta^{2}\right)
$$


where

$$
\beta=\frac{\sqrt{\pi}}{2 R^{\prime}} \frac{\Gamma\left(\frac{1}{2 \sigma}+\frac{1}{2}\right)}{\Gamma\left(\frac{1}{2 \sigma}+1\right)},
$$

and $R^{\prime}$, which by (2.9) is equal to $R$ in zeroth-order, will be chosen later. Next, $\varrho$ is scaled by

$$
\varrho=\frac{\bar{\varrho}}{\alpha}
$$

where

$$
\alpha=\sqrt{\pi \sigma}\left(\sqrt{2}(\sigma+1)^{\sigma / 2} \frac{\Gamma\left(\frac{1}{2 \sigma}\right)}{\Gamma\left(\frac{1}{2 \sigma}+\frac{1}{2}\right)} R^{\prime \prime}\right)^{\frac{-1}{\sigma+1}} .
$$

Here $R^{\prime \prime}$ is defined by the condition that the boundary $B$ intersects the plane $z=0$ at $\varrho=1 / \alpha$. It follows from the last remark in Sect. 2 that $R^{\prime \prime}$ is also equal to $R$ in zerothorder. Instead of $\varrho$ it is more convenient to use the variable $\tau$ [see $(4.22)$ of I] defined by

$$
\tau=\frac{\bar{\varrho}}{r(\zeta)},
$$

where the boundary $B$ is given by $\bar{\varrho}_{b}=r(\zeta)$. We now define the expansion parameter $\Lambda$ in terms of $\alpha$ and $\beta$ by

$$
\Lambda=\frac{8 \alpha^{2}}{\beta^{2}}=\frac{4}{\sigma}\left(\frac{\sigma+1}{2}\right)^{\frac{-\sigma}{\sigma+1}}\left(\frac{\Gamma\left(\frac{1}{2 \sigma}\right)}{\Gamma\left(\frac{1}{2 \sigma}+\frac{1}{2}\right)}\right)^{\frac{2 \sigma}{\sigma+1}} R^{\prime 2} R^{\prime \prime \frac{-2}{\sigma+1}} .
$$

Finally, we rescale $\Psi$ by

$$
\Psi=\left(\frac{R^{\prime \prime}}{R^{\prime}}\right)^{\frac{2}{\sigma}} \psi
$$

[For the special case $\sigma=1$ treated in I, $R^{\prime}$ and $R^{\prime \prime}$ are respectively $\Lambda^{\prime} / 4 \sqrt{\pi}$ and $\Lambda^{\prime \prime} / 4 \sqrt{\pi}$, while the $\Lambda$ of (4.6) reduces to that of (5.9) of I.]

For the purpose of obtaining the first-order solution, we begin with (2.2) written as:

$$
\begin{aligned}
\frac{g(D)}{D} & {\left[\left(\partial_{\varrho} \Psi\right)^{2} \partial_{z z} \Psi+\left(\partial_{z} \Psi\right)^{2} \partial_{\varrho \varrho} \Psi-2\left(\partial_{z} \Psi\right)\left(\partial_{\varrho} \Psi\right)\left(\partial_{z \varrho} \Psi\right)\right] } \\
& +\left[\left(\partial_{z} \Psi\right)^{2}+\left(\partial_{\varrho} \Psi\right)^{2}\right]\left[\partial_{z z} \Psi+\partial_{\varrho \varrho} \Psi-\frac{1}{\varrho} \partial_{\varrho} \Psi\right]=0 .
\end{aligned}
$$


To exhibit the dependence of $g(D) / D$ on $\Lambda$, we define $\bar{D}$ by

$$
\bar{D}=\frac{\pi \sigma}{(\sigma+1) \alpha^{2}}\left(\frac{R^{\prime \prime}}{R^{\prime}}\right)^{\frac{2}{\sigma}} D .
$$

In terms of this $\bar{D}$,

$$
\frac{g(D)}{D}=\frac{\Lambda}{4} \bar{D}^{-\sigma} h\left(\frac{4}{\sigma \Lambda} \bar{D}^{\sigma}\right)
$$

where

$$
h(x)=1+\left(1-\sigma-2 b_{2}\right) x+\ldots .
$$

Carrying out the change of variables, we obtain from (4.8) and (4.9)

$$
\begin{aligned}
2 \bar{D}^{-\sigma} h\left(\frac{4}{\sigma \Lambda} \bar{D}^{\sigma}\right)\left(1-\zeta^{2}\right)^{1-\frac{1}{\sigma}}\left\{\left(\partial_{\zeta} \psi\right)^{2} \partial_{\tau \tau} \psi+\left(\partial_{\tau} \psi\right)^{2}\left[2 \frac{\partial_{\zeta} r}{r} \partial_{\zeta} \psi-\tau \frac{\partial_{\zeta \zeta} r}{r} \partial_{\tau} \psi\right.\right. \\
\left.\left.+\partial_{\zeta \zeta} \psi-\frac{(\sigma-1) \zeta}{\sigma\left(1-\zeta^{2}\right)} \partial_{\zeta} \psi+\frac{(\sigma-1) \zeta}{\sigma\left(1-\zeta^{2}\right)} \tau \frac{\partial_{\zeta} r}{r} \partial_{\tau} \psi\right]-2\left(\partial_{\zeta} \psi\right)\left(\partial_{\tau} \psi\right)\left(\partial_{\zeta \tau} \psi\right)\right\} \\
+\left(\partial_{\tau} \psi\right)^{2}\left[1+\frac{8}{\Lambda}\left(1-\zeta^{2}\right)^{1-\frac{1}{\sigma}}\left(\tau \partial_{\zeta} r-r \frac{\partial_{\zeta} \psi}{\partial_{\tau} \psi}\right)^{2}\right]\left\{\frac{1}{r^{2}} \tau \partial_{\tau}\left(\frac{\partial_{\tau} \psi}{\tau}\right)\right. \\
+\frac{8}{\Lambda}\left(1-\zeta^{2}\right)^{1-\frac{1}{\sigma}}\left[\left(\frac{\partial_{\zeta} r}{r}\right)^{2} \tau \partial_{\tau}\left(\tau \partial_{\tau} \psi\right)-2 \tau \frac{\partial_{\zeta} r}{r} \partial_{\zeta \tau} \psi-\tau \partial_{\zeta}\left(\frac{\partial_{\zeta} r}{r}\right) \partial_{\tau} \psi\right. \\
\left.\left.+\partial_{\zeta \zeta} \psi-\frac{(\sigma-1) \zeta}{\sigma\left(1-\zeta^{2}\right)} \partial_{\zeta} \psi+\frac{(\sigma-1) \zeta}{\sigma\left(1-\zeta^{2}\right)} \tau \frac{\partial_{\zeta} r}{r} \partial_{\tau} \psi\right]\right\}=0,
\end{aligned}
$$

with

$$
\bar{D}=\frac{-\sigma}{2(\sigma+1)} \frac{\partial_{\tau} \psi}{\tau r^{2}}\left[1+\frac{8}{\Lambda}\left(1-\zeta^{2}\right)^{1-\frac{1}{\sigma}}\left(\tau \partial_{\zeta} r-r \frac{\partial_{\zeta} \psi}{\partial_{\tau} \psi}\right)^{2}\right]^{1 / 2} .
$$

The partial differential Eq. (4.12), which is the generalization of (5.10) of I, is exact.

\section{First-Order Solution}

To first order in $\Lambda^{-1}$,

$$
r(\zeta)=\left(1-\zeta^{2}\right)^{\frac{1}{2 \sigma}}\left[1+\frac{1}{\Lambda} w(\zeta)\right] .
$$

The fact that (4.12) is homogeneous in $\psi$ simplifies the perturbation series of $\psi(\zeta, \tau)$. To first order the implication is that

$$
\psi(\zeta, \tau)=\left(1-\tau^{2}\right)^{\frac{\sigma+1}{\sigma}}\left\{1+\frac{1}{\Lambda}\left[c+\tau^{2} k(\zeta)\right]\right\} .
$$

In (5.1) and (5.2) $w$ and $k$ are even functions of $\zeta$. The zeroth-order solution (2.11) has been used. Because of the boundary condition at $\tau=0$,

$$
\psi(\zeta, 0)=\left(\frac{R^{\prime}}{R^{\prime \prime}}\right)^{\frac{2}{\sigma}}=1+\frac{c}{\Lambda},
$$

$c$ does not depend on $\zeta$. 
The substitution of (5.1) and (5.2) into (4.12) yields two ordinary differential equations for $w(\zeta)$ and $k(\zeta)$ :

$$
\sigma\left(1-\zeta^{2}\right)^{2} w^{\prime \prime}-\zeta(\sigma+1)\left(1-\zeta^{2}\right) w^{\prime}-2(\sigma+1) w+\sigma(c+k)+\frac{4(\sigma+4) \zeta^{2}}{\sigma^{2}\left(1-\zeta^{2}\right)}=0
$$

and

$$
\begin{aligned}
& \sigma\left(1-\zeta^{2}\right)^{2} k^{\prime \prime}-\zeta(\sigma+1)\left(1-\zeta^{2}\right) k^{\prime}-4(2 \sigma+1) k-\frac{8(\sigma+1)\left(1-2 b_{2}\right)}{\sigma^{2}\left(1-\zeta^{2}\right)} \\
& -\frac{32(\sigma+1)^{2} \zeta^{2}}{\sigma^{3}\left(1-\zeta^{2}\right)}=0
\end{aligned}
$$

with the boundary condition $w(0)=0$.

The solution of $(5.5)$ is

$$
\begin{aligned}
k(\zeta)= & \frac{2(\sigma+1)}{\sigma^{2}(\sigma+3)}\left\{\frac{2 \sigma-1}{2 \sigma+1}\left(1-2 b_{2}\right)+\frac{4(\sigma+1)(3 \sigma+2)}{\sigma(2 \sigma+1)}\right. \\
& \left.-2\left[1-2 b_{2}+\frac{4(\sigma+1)}{\sigma}\right] \frac{1}{\left(1-\zeta^{2}\right)}\right\} .
\end{aligned}
$$

The solution of (5.4) is more subtle. The following two points are relevant. First, this equation involves the constant $c$ which we are at liberty to choose by varying $R^{\prime}$ as seen from (5.3). Secondly, the even solution of the homogeneous equation is transcendental. Therefore, the solution of (5.4) can be elementary only for a special value of $c$. This particular value of $c$ can be found with the ansatz

$$
w(\zeta)=\frac{K \zeta^{2}}{1-\zeta^{2}}
$$

The substitution of (5.6) and (5.7) into (5.4) leads to a linear equation in $\zeta^{2}$. This determines both $K$ and $c$ :

$$
\begin{gathered}
K=\frac{2}{\sigma^{2}(\sigma-1)(\sigma+3)}\left[-\sigma(\sigma+1) b_{2}+2 \sigma^{2}+\sigma-4\right], \\
c=\frac{2}{\sigma^{2}(\sigma-1)(\sigma+3)(2 \sigma+1)}\left[6(\sigma+1) b_{2}-2 \sigma^{3}-\sigma^{2}+8 \sigma+1\right] .
\end{gathered}
$$

This first-order solution is given by (5.1) and (5.2) together with (5.6)-(5.9). Unfortunately, this elementary solution is not completely satisfactory because it fails to reduce to our previous solution given in I for $\sigma \rightarrow 1$. Indeed, in this limit both $K$ and $c$ approach infinity.

We proceed to give an alternative choice of $c$ which avoids this difficulty. First, the even solution of the homogeneous equation corresponding to (5.4) is

$$
w_{0}(\zeta)=1+\frac{\sigma+1}{\sigma} \zeta^{2} \cdot\left(1-\zeta^{2}\right)^{-\frac{\sigma+1}{2 \sigma}} F\left(-\frac{1}{2 \sigma}+\frac{1}{2}, \frac{1}{2} ; \frac{3}{2} ; \zeta^{2}\right) \text {. }
$$

In the limit $\sigma \rightarrow 1$ this $w_{0}$ is simply

$$
w_{0} \rightarrow \frac{1+\zeta^{2}}{1-\zeta^{2}}
$$


Since $w_{0}(0)=1$ and $w(0)=0$ for all $\sigma$, we look for a solution of the form

$$
w(\zeta)=\mathrm{const}\left[\frac{1+\tilde{\alpha} \zeta^{2}}{1-\zeta^{2}}-w_{0}(\zeta)\right],
$$

where $\tilde{\alpha}=\tilde{\alpha}(\sigma)$ must satisfy $\tilde{\alpha}(1)=1$. Aside from this condition, $\tilde{\alpha}(\sigma)$ is arbitrary. Therefore, there are many solutions. We have found that a convenient choice is

$$
\tilde{\alpha}(\sigma)=\frac{1}{\sigma} .
$$

With this choice, it follows from (5.4) that $w$ and $c$ are given by

$$
\begin{gathered}
w=\frac{2}{\sigma^{2}(\sigma-1)(\sigma+3)}\left[-\sigma(\sigma+1) b_{2}+2 \sigma^{2}+\sigma-4\right] \frac{\zeta^{2}}{1-\zeta^{2}} \\
\cdot\left[1-\left(1-\zeta^{2}\right)^{\frac{\sigma-1}{2 \sigma}} F\left(-\frac{1}{2 \sigma}+\frac{1}{2}, \frac{1}{2} ; \frac{3}{2} ; \zeta^{2}\right)\right], \\
\quad c=\frac{2(\sigma+1)}{\sigma^{2}(\sigma+3)(2 \sigma+1)}\left[-2(2 \sigma+3) b_{2}+6 \sigma+7\right] .
\end{gathered}
$$

Here (5.14) and (5.15) replace (5.7)-(5.9).

It is seen from either (5.8) or (5.14) that the boundary $B$ remains unchanged to first order when

$$
b_{2}=\frac{2 \sigma^{2}+\sigma-4}{\sigma(\sigma+1)} .
$$

This is the generalization of the phenomenon found in I for the case $b_{2}=-1 / 2$.

The two versions of the first-order solution correspond to different choices of $R^{\prime}$. By (4.1) and (4.2) a different choice of $R^{\prime}$ implies a redefinition of the variable $\zeta$ which amounts to a non-elementary transformation between the old and the new $\zeta$.

Acknowledgements. One of us (T.T.W.) wishes to thank Professor Fritz Gutbrod, Professor Hans Joos, Professor Paul Söding, and Professor Volker Soergel for their kind hospitality at DESY.

\section{References}

1. Lehmann, H., Wu, T.T.: Nucl. Phys. B 237, 205 (1984)

2. Pagels, H., Tomboulis, E.: Vacuum of the quantum Yang-Mills theory and magnetostatics. Nucl. Phys. B 143, 485 (1978)

3. Adler, S.L.: Effective-action approach to mean-field non-Abelian statics and a model for bag formation. Phys. Rev. D 23, 2905 (1981)

Adler, S.L., Piran, T.: Flux confinement in the leading logarithm model. Phys. Lett. 113B, 405 (1982)

Adler, S.L., Piran, T.: Relaxation methods for gauge field equilibrium equations. Rev. Mod. Phys. 56, 1 (1984)

4. Gelfand, I.M., Shilov, G.E.: Generalized functions, Vol. 1. New York: Academic Press, 1964

Communicated by G. Mack

Received June 27, 1984 
